\title{
A Platform for Self-Management Supported by Assistive, Rehabilitation and Telecare Technologies
}

\author{
R. J. Davies, L. B. Galway, C. D. Nugent, C. H. Jamison, R. E. Gawley, P. J. McCullagh, H. Zheng, N. D. Black \\ School of Computing and Mathematics and Computer Science Research Institute \\ University of Ulster \\ Shore Road, Newtownabbey \\ Co. Antrim, BT37 0QB
}

\{rj.davies, 1.galway, cd.nugent, ch.jamison, re.gawley, pj.mccullagh, h.zheng, nd.black\}@ulster.ac.uk

\begin{abstract}
Long-term chronic conditions pose a significant challenge in modern healthcare systems in terms of a reduction in quality of life and the increased costs associated with their management. The concept of self-management can help address these issues supported through the introduction of home based assistive and rehabilitation telecare technologies. The proposed solution aims to produce a home-based platform that supports a number of chronic diseases such as Chronic Pain, Congestive Heart Failure and Stroke. The long-term vision of this work is to take into consideration supporting further conditions such as Chronic Obstructive Pulmonary Disease. The system aims to promote behavior change by monitoring activity metrics, performing analysis, which ultimately leads to providing feedback to the user in a meaningful and engaging manner.
\end{abstract} System

Telecare; Long-Term Chronic Conditions; Self-Management

\section{INTRODUCTION}

In order to empower patients suffering from chronic conditions with the ability to self-manage their recuperation and rehabilitation, modern computing and health-based sensor technologies may be used to produce a telecare system capable of supporting a range of conditions. This paper aims to present an overview of a telecare system, entitled "Self-Management Supported by Assistive, Rehabilitation and Telecare Technologies" (SMART2 ${ }^{l}$ ), which has been designed to facilitate those suffering from clinical conditions, including Chronic Pain $(C P)$, Congestive Heart Failure $(C H F)$ and Stroke. Although the overarching implementation and subsequent realistic evaluation of the SMART2 system is currently ongoing, this paper focuses on presenting the technical details of the system architecture. The remainder of this paper is presented as follows: a description of the infrastructure and key technical components of the SMART2 system are detailed in Section II, followed by an outline of future work in Section III.

\section{SMART2 SYSTEM ARCHITECTURE}

To realize the design of a system that facilitates assistive and rehabilitative support for the self-management of a range of clinical conditions, an appropriate set of technologies is

\footnotetext{
$1 \mathrm{http}: / /$ www.thesmartconsortium.org/Smart_2/index.php
}

required. These included technologies pertinent to the overall operation of the system, namely home and mobile computing platforms, along with technologies specific to the clinical conditions, such as sensor-based insoles and wireless digital scales. Figure 1 provides an illustration of the primary hardware technologies utilized within the SMART2 system according to three preselected conditions.

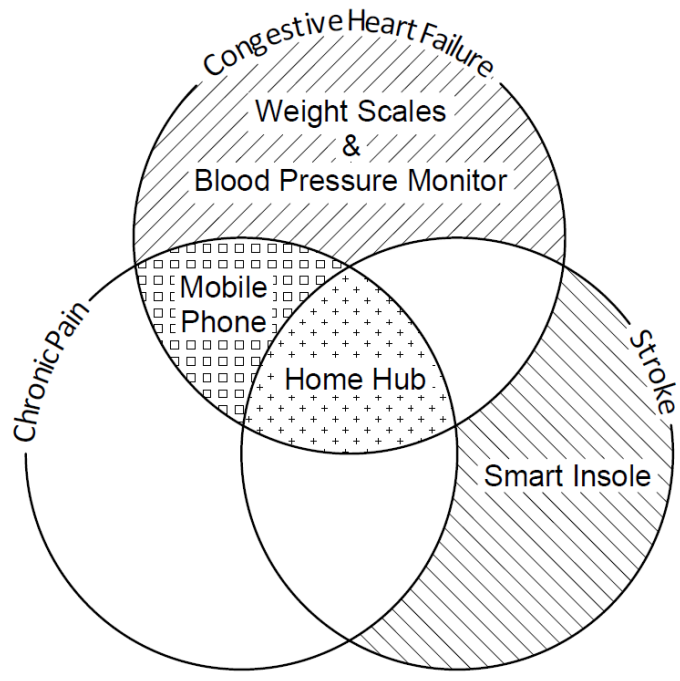

Figure 1. Overview of SMART2 system technologies by clinical condition.

As depicted in Figure 1, the central component of the SMART2 system is a home computing platform, referred to as the Home Hub, which is independent of the conditions supported and works in conjunction with other system components in order to provide the self-management capabilities. To tailor the system for users suffering from $\mathrm{CP}$, the Home Hub is coupled with a mobile computing platform, referred to as the Mobile Phone. This platform acts as a customized, personal activity monitor for the user, permitting activity-related information to be obtained and wirelessly transmitted to the Home Hub for further analysis. The Home Hub is used in conjunction with a Mobile Phone, Digital Scales and a Digital Blood Pressure Monitor in order to configure the system for users suffering from CHF. For users of the system suffering from Stroke, a pair of wireless sensor-based insoles, 
known as the Smart Insole, is used alongside the Home Hub in order to capture activity-related foot pressure information and provide subsequent gait analysis. Further details of the key components of the system, notably the Home Hub, Mobile Phone and Smart Insole are presented in the following Sections.

\section{A. Home Hub}

The Home Hub is the central technical component of the SMART2 system and supports the primary functions for user interaction, user engagement, general processing and operation as a central communication component. In terms of hardware technology the Home Hub consists of an all in one computer with a multi touch screen. It features a dual core $1.6 \mathrm{GHz}$ processor, 2 GB RAM, 500 GB hard disk and a high definition 1600x900 multi-touch touch screen [1]. Figure 2 provides an overview of the SMART2 system, depicting the role the Home Hub plays within the overall infrastructure.

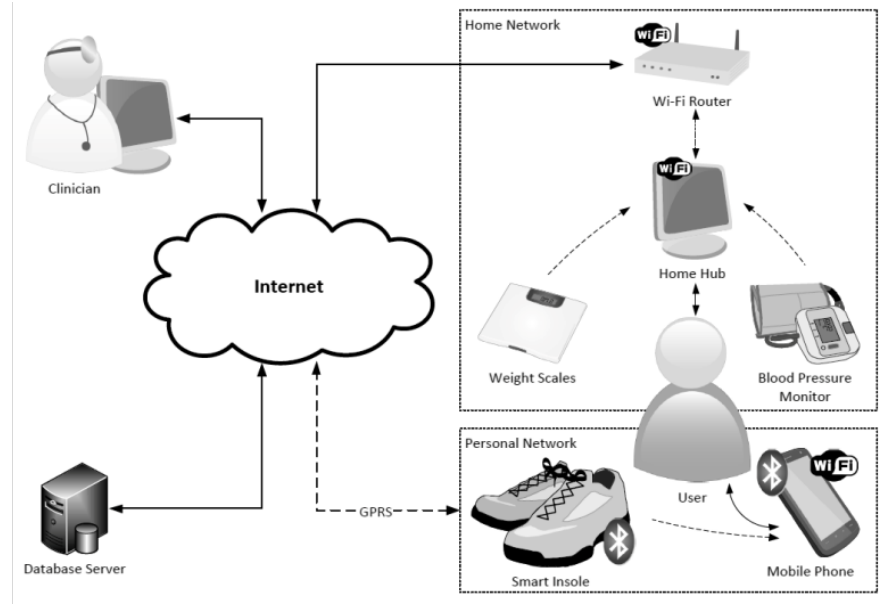

Figure 2. Overview of SMART2 system infrastructure.

The main function of the Home Hub is to encourage user engagement. To help facilitate user engagement, graphical components developed using Adobe Flash drive the Graphical User Interface (GUI) of the system, thereby providing an interface that is both simplistic and feature-rich. By utilizing touch screen technology as the primary mechanism for user navigation, all user interaction is conducted through intuitive touch gestures. Subsequently, it is anticipated that this approach to the design and implementation of the GUI for the Home Hub will yield a high level of engagement and usability for the user within a short degree of exposure to the system. As previously discussed, there are three instantiations of the Home Hub, in terms of the software delivered to the user, however, there is a strong correlation between each of the conditions. A common framework and graphical theme has been adopted in order to maintain coherency between each of the three systems. In terms of development, this strategy promotes extensibility through component reuse.

The Home Hub provides mechanisms for information processing and communications with the other physical components of the system, such as the Database Server, Mobile Phone and peripheral devices, as illustrated in Figure 2. For the implementation of these subsystems, the Java programming platform (JDK 1.6) was utilized, featuring TCP/IP socket technology combined with a customized XML protocol to facilitate message passing. Through periodically conducting analysis following data collection from the peripheral devices, the Home Hub has the ability to provide users with an enhanced level of feedback. Additionally, as Figure 2 shows, there is also a duplex communications channel between the Mobile Phone and the Database Server, via the Home Hub, in order to permit access to user-specific data. Such data is subsequently utilized to deliver user-specific content on the Mobile Phone.

\section{B. Mobile Phone}

Although the main point of interaction and information delivery between users and the SMART2 system will be the Home Hub, users suffering from CP or CHF will also utilize a Mobile Phone in order to select activities, capture pedometer and location-based data, in addition to acting as a mechanism to provide feedback on the activities performed. The HTC HD2 phone [2], featuring a $1 \mathrm{GHz}$ processor, 448MB RAM, 480x800 Wide Video Graphics Array capacitive touch screen, internal accelerometer and hardware GPS module has been selected as the Mobile Phone for users suffering from both $\mathrm{CP}$ and $\mathrm{CHF}$ conditions. Given that the HD2 runs on the Windows Mobile 6.5 Professional platform, the mobile application software was developed in C\# using the .NET Compact Framework 3.5. Figure 3 presents the details of the data-flow throughout the key components of the mobile application, as configured for users suffering from the CP condition.

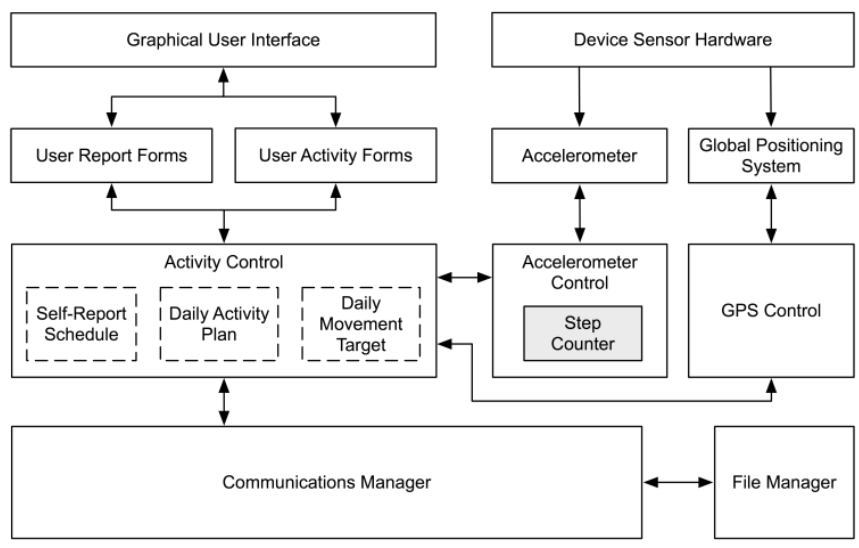

Figure 3. Mobile application data flow for CP (Step Counter highlighted).

One of the key components of the mobile application as shown in Figure 3 is the Activity Control, which is responsible for maintaining the selection and completion of daily activities, daily activity targets and scheduled user self-reports. Through the Activity Control, the mobile application presents the appropriate screens to the user and acquires both explicit and implicit user data from the touch screen and device sensor hardware respectively. The Activity Control communicates extensively with the Communications Manager component of the application in order to initiate and complete communication requests with the Home Hub. One of the main purposes of such requests is to periodically synchronize the Mobile Phone with the Home Hub by obtaining the daily goal activities, daily activity targets and the schedule for self-reports. Similarly, 
another primary purpose of the communications requests is to periodically submit activity-related data and user reports to the Home Hub for further analysis, display and storage. The Communications Manager facilitates message passing with the Home Hub using a customized XML protocol generated and parsed by the File Manager component of the application. As Figure 3 shows, the other key components that interoperate with the Activity Control are the Accelerometer Control and the GPS Control, which are utilized to manipulate the Mobile Phone's accelerometer and GPS respectively. For both the CP and CHF variants of the SMART2 system, the Accelerometer Control provides one of the primary mechanisms for the generation of user activity metrics. Throughout the lifetime of the mobile application, the Activity Control regularly determines the active step count of the user through the Step Counter subcomponent of the Accelerometer Control.

Implementation of the algorithm utilized by the Step Counter features two modes of operation: (1) threshold parameter configuration and (2) normal pedometer operation. The flowchart presented in Figure 4 highlights the various high-level decisions made during normal pedometer operation.

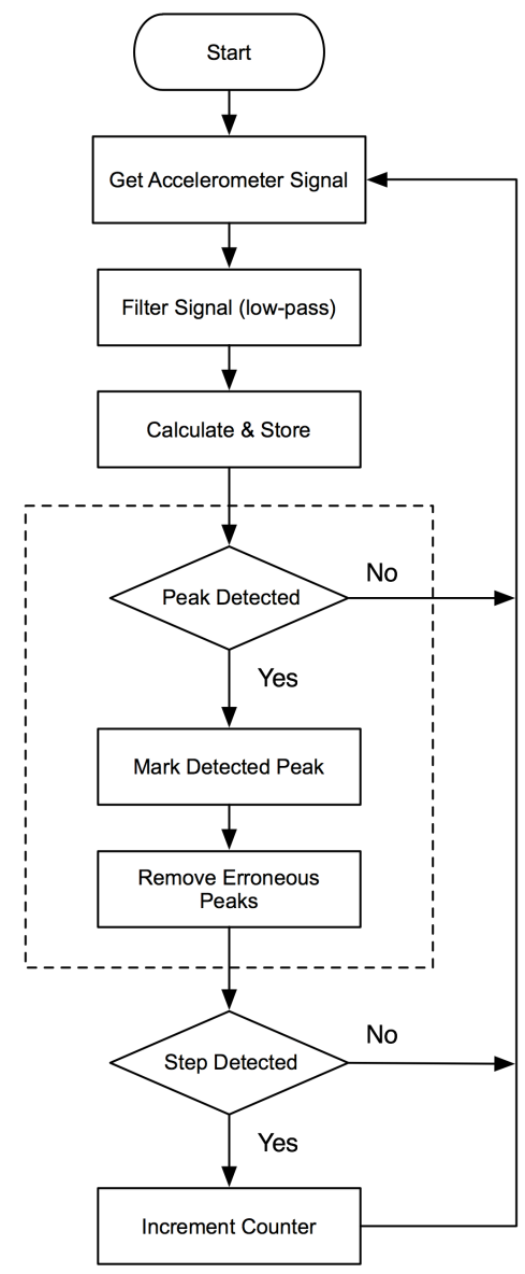

Figure 4. Normal pedometer operation of the step counter algorithm.

Based upon the research presented in [3] [4], the algorithm has been extended for real-time operation on the Mobile Phone, operating at a sampling frequency of $10 \mathrm{~Hz}$ with a $20 \mathrm{~Hz}$ lowpass filter.

\section{Smart Insole}

The Smart Insoles are utilized to capture information such as plantar pressure data and weight for the Stoke cohort. A "WalkinSense" device from Tomorrow Options [5] has been utilized to capture individual foot pressure data, number of steps taken, distance walked and walking speed. Each device consists of an Information Collection and Processing Unit with $1 \mathrm{~GB}$ of memory and eight pressure sensors, which may be placed anywhere on the insole, thereby accommodating any foot size. The position of the sensors also facilitates gait analysis, which is useful for monitoring functional recovery of users suffering from Stroke [6]. During everyday activities, the data acquired by the insoles is streamed over Bluetooth to a mobile device, which in turn relays the data to the Home Hub via Wi-Fi, as previously shown in Figure 2. Upon receipt of the data, the Home Hub performs gait analysis and provides a visual indication of post-activity feedback to the user. By utilizing a mobile device in conjunction with the Smart Insoles the maximum freedom is offered to the user by enabling plantar pressure data to be captured both inside and outside the home environment.

\section{CONCLUSION}

Utilizing a range of current personal computing and healthcare technologies, it is feasible to design and develop a telecare system supporting the self-management of a range of chronic conditions. This paper presents an overview of such a system, outlining the infrastructure and key components therein. Although the system is currently under development, it is anticipated that it will be further enhanced based on feedback obtained from realistic user evaluation, which will be conducted across the three conditions of $\mathrm{CP}, \mathrm{CHF}$ and Stroke. Additionally, further research may be conducted by extending the system to facilitate other chronic conditions.

\section{ACKNOWLEDGMENT}

This research has been supported by the Engineering and Physical Sciences Research Council, UK (EP/F001916).

\section{REFERENCES}

[1] ASUS Eeetop PC ET2010PNT Specifications, accessed March 2011. http://uk.asus.com/product.aspx?P_ID=nAjcDr6gLlY51f6e

[2] HTC HD2 Specifications, accessed Feb 2011; http://www.htc.com/europe/product/hd2/specification.html

[3] H. Chan, H. Zheng, H. Wang, R. Gawley, M. Yang, and R. Sterrit, "Feasibility Study on iPhone Accelerometer for Gait Analysis," in Proc $5^{\text {th }} \quad$ International ICST Conference on Pervasive Computing Technologies for Healthcare (in press). Dublin, Ireland, May 2011

[4] Y. Huang, H. Zheng, C. Nugent, P. McCullagh, S. McDonough, M. Tully, and S. O'Connor, "Activity monitoring using an intelligent mobile phone - a validation study", in ACM Proc. the 3rd International Conference on Pervasive Technologies Related to Assistive Environments. Samos, Greece, June 2010

[5] Tomorrow Options, accessed Feb 2011; http://www.tomorrowoptions.com/en/walkinsense

[6] H. P. von Schroeder, R. D. Coutts, P. D. Lyden and E. Billings, Jr, "Gait parameters following stroke: a practical assessment." J Rehabil Res Dev 32, 1995, pp. 25-31. 